\title{
Phyllosilicate fabric characterization by Low-Temperature Anisotropy of Magnetic Susceptibility (LT-AMS)
}

\author{
Josep M. Parés and Ben A. van der Pluijm \\ Dept. of Geological Sciences, University of Michigan, Ann Arbor, MI, USA
}

Received 10 May 2002; revised 23 August 2002; accepted 27 August 2002; published 27 December 2002.

[1] This paper examines the use and applicability of anisotropy of magnetic susceptibility at Low-Temperature $\left(77^{\circ} \mathrm{K}\right)$, called LT-AMS. Paramagnetic susceptibility increases significantly with low temperature, which permits high precision determination of the anisotropy of paramagnetic susceptibility of a rock from measurements at liquid nitrogen temperature. We describe a new procedure that measures directional susceptibility in an oriented rock specimen while immersed in liquid nitrogen. Our procedure overcomes the problem of temperature heterogeneity within a rock sample while its susceptibility is being measured, offering the opportunity to measure low-temperature susceptibility in multiple orientations. This non-destructive method provides a way of characterizing the principal paramagnetic minerals present in a rock, based on temperature-induced changes of the anisotropy degree. Moreover, the method offers a better-resolved relationship between anisotropy degree and grain alignment, which is used to quantify rock fabrics. INDEX TERMS: 1518 Geomagnetism and Paleomagnetism: Magnetic fabrics and anisotropy; 1540 Geomagnetism and Paleomagnetism: Rock and mineral magnetism; 1594 Geomagnetism and Paleomagnetism: Instruments and techniques; 5109 Physical Properties of Rocks: Magnetic and electrical properties; 8030 Structural Geology: Microstructures. Citation: Parés, J. M., and B. A. van der Pluijm, Phyllosilicate fabric characterization by LowTemperature Anisotropy of Magnetic Susceptibility (LT-AMS), Geophys. Res. Lett., 29(24), 2215, doi:10.1029/2002GL015459, 2002.

\section{Introduction}

[2] The fabric of a rock includes the complete spatial and geometric configuration of all its components and encompasses (micro)structure and crystallographic preferred orientation of the ensemble of minerals. An intrinsic property of most rock-forming minerals is that magnetic susceptibility, the ratio of magnetization to the applied field, is anisotropic [Nye, 1957], and can potentially be used to delineate and characterize components to the rock fabric. The total magnetic susceptibility of a rock is given by the bulk contribution of its mineralogy, including paramagnetic (e.g., phyllosilicates, ferromagnesians, iron-bearing feldspars), diamagnetic (e.g., quartz, calcite) and ferromagnetic (e.g., magnetite, hematite) grains. In addition, fluids filling any porosity will contribute, to a lesser extent, to the total bulk susceptibility of a rock. The Anisotropy of Magnetic Susceptibility (AMS) in rocks mostly depends on crystallographic preferred orienta- tion, compositional layering, distribution and size of microfractures and the shape fabric of grains, which may interact in complex ways [see Tarling and Hrouda, 1993].

[3] AMS has been successfully used to investigate the spatial and geometric configuration of rock components and for qualitative estimation of fabric development in a variety of geologic environments (see Housen and van der Pluijm, 1991; Tarling and Hrouda, 1993 and Borradaile and Henry, 1997 and Parés et al., 1999 for references).

[4] AMS of most rocks is typically determined by standard low-field systems (e.g. Kappabridge, Bartington, Sapphire instruments). However, the concentration and mineralogy of the phases responsible for AMS can hamper the successful application of the method in many rock types. In some cases the mineralogy is not favorable, because of the presence of large amounts of diamagnetic or ferromagnetic grains that overwhelm the paramagnetic contribution. In other cases, the rock fabric is too weak or is ill-defined to satisfactorily apply standard AMS measurements. In most rocks [see Tarling and Hrouda, 1993 for a review], the AMS is mostly carried by paramagnetic phases, and specifically phyllosilicates, that often carry critical information on the geologic history. In these circumstances, a method that enhances the resolution of the susceptibility tensor would be highly desirable, which is the focus of this paper.

[5] Among the methods to enhance AMS are the so-called "high temperature methods", which rely on mineralogic and chemical breakdown reactions of phyllosilicates and ferromagnetic phases when heated above a certain temperature. [Urrutia-Fucugauchi and Tarling, 1983; Perarnau and Tarling, 1985; Schultz-Krutisch and Heller, 1985; Xu et al., 1991; Borradaile and Lagroix, 2000]. Heating typically results in new minerals that often mimic the crystallographic structure of micas and clays, the major carriers of AMS, resulting in a better definition of magnetic anisotropy. Major drawbacks of high-temperature methods include the destructive nature and that any changes in magnetic mineralogy and petrology must be monitored.

[6] Low-Temperature (LT) methods have been used to an alternative approach to heating samples to enhance bulk magnetic susceptibility. As opposed to heating, the basis of LT methods is the strong dependence of paramagnetic susceptibility on temperature, which, as shown below, involves no irreversible changes to the phases contributing to susceptibility.

[7] LT methods, based on warming curves from $77^{\circ} \mathrm{K}$ to room temperature, have been used to separate para- and ferromagnetic bulk contributions in rocks [Schultz-Krutisch and Heller, 1985; Jover et al., 1989; Richter and van der Pluijm, 1994] but, with the exception of the studies by Ihmlé et al. [1989] and Lüneburg et al. [1999], never to determine the anisotropy of LT susceptibility. 
[8] Here, we describe a new method for determining magnetic fabrics at $77^{\circ} \mathrm{K}$ for selected rocks that enhances and refines the anisotropy of paramagnetic susceptibility tensor. Other methods that isolate paramagnetic fabric are based on the increase in induced magnetization of paramagnetic and diamagnetic minerals as applied field is increased [Parma, 1988; Rochette and Fillion, 1988; Hrouda and Jelinek, 1990; Bergmuller et al., 1994; Borradaile and Werner, 1994; Martín-Hernández and Hirt, 2001]. Notably, the magnetization of ferromagnetic minerals saturates at particular field strengths. Recently, high-field measurements carried out on a VSM have also been used to calculate the high-field AMS ellipsoid parameters [Thill et al., 2000; Ferré et al., 2000]. However, these methods require sophisticated rock magnetic instrumentation that is not readily available to most workers and is relatively time-consuming, as opposed to the relatively simple yet reliable method we present.

\section{Background}

[9] Paramagnetic susceptibility in sheet silicates is related to the presence of transition metal ions, typically iron. If iron in the silicates is sufficiently dilute, magnetic interactions, which tend to align the moments of neighboring cations, may be neglected. In this case, magnetic susceptibility follows a Curie law [Morrish, 1965], $\mathrm{x}_{\mathrm{p}}=\mathrm{C} / \mathrm{T}$, where $\mathrm{x}_{\mathrm{p}}$ is the mass susceptibility, $\mathrm{C}$ is the Curie constant and $\mathrm{T}$ the absolute temperature. At high concentrations of iron, magnetic interactions can no longer be neglected, because there might be magnetic cations in adjacent sites where their spins are coupled by exchange interactions. The susceptibility then follows the Curie-Weiss law, $\mathbf{x}_{\mathrm{p}}=\mathrm{C} /(\mathrm{T}-\theta)$, where $\theta$ is the paramagnetic Curie temperature. Plots of inverse susceptibility versus temperature give the paramagnetic Curie temperature, $\theta$. If $\theta$ is negative, the material is antiferromagnetic; if positive, it is ferromagnetic; $\theta$ is zero for paramagnetic materials [see Dunlop and Ozdemir, 1997 for details]. As the magnetic-ordering transition temperature is approached from above $T_{C}$ or $T_{N}$, the paramagnetic susceptibility, $X_{p}$, increases considerably. The increase of susceptibility at low temperature is unequal through sheet iron-silicates: The magnetic order behaves in a ferromagnetic manner within the basal plane, but as antiferromagnetic one between planes [Beausoleil et al., 1983] and the ratio of susceptibility parallel and perpendicular to the basal plane increases at low temperature.

\section{Measurement of Magnetic Susceptibility as a Function of Temperature}

[10] A procedure for evaluating the relative contribution of paramagnetic and ferromagnetic phases is given by the method of Richter and van der Pluijm [1994]. Here we use a SI2B susceptibility meter with an internal coil frequency of $19.2 \mathrm{kHz}$ (Sapphire Instruments). The advantage of using a large coil (SI2B, internal diameter $45 \mathrm{~mm}$ ) is two-fold: (1) susceptibility-temperature curves can be obtained from different orientations of the same specimen and (2) standard paleomagnetic samples $(2.11 \mathrm{~cm}$ length $\times 2.54 \mathrm{~cm}$ diameter) can be used [Parés et al., 2000].

[11] The examples used in this study comprise a range of micaceous rocks (siltstones, sandstones, slates and schists)
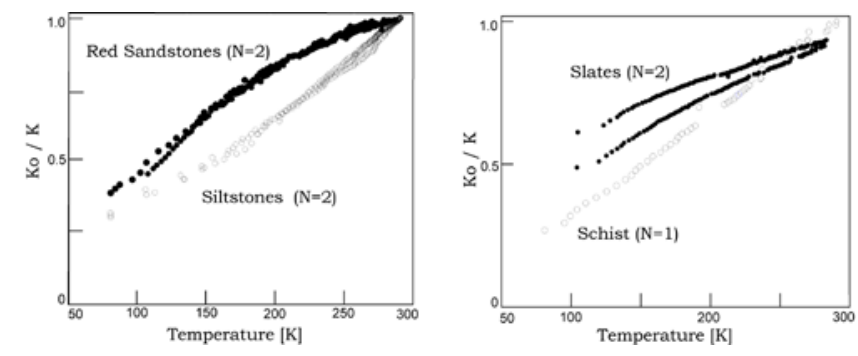

Figure 1. Temperature dependence of low-field susceptibility for samples of selected rocks. $\mathrm{K}_{0} / \mathrm{K}$ is the normalized reciprocal magnetic susceptibility (temperature during warming relative to ambient temperature, $290^{\circ} \mathrm{K}$ ). The increase in reciprocal susceptibility between $100^{\circ} \mathrm{K}$ and $290^{\circ} \mathrm{K}$ indicates that samples are dominated by paramagnetic minerals. N- number of samples.

with different relative phyllosilicate content and mineralogy. The results of $\mathrm{k}(\mathrm{T})$ curves allow us to evaluate the relative content of paramagnetic phases (Figure 1), and reveal that the magnetic susceptibility increases as temperature decreases, as predicted by the Curie-Weiss Law. The concave upwards shape of the curve for red sandstones is indicative of a small ferromagnetic contribution. Otherwise, the susceptibility of all studied samples is dominantly paramagnetic and all obey the Curie-Weiss Law.

\section{Low-Temperature Anisotropy of Magnetic Susceptibility}

[12] We aim to extend the obvious potential of nondestructive, LT bulk susceptibility measurements, by determining the LT AMS of clay-bearing rocks. Determination of LT AMS involves measurement of directional susceptibilities at liquid nitrogen temperature $\left(77^{\circ} \mathrm{K}\right)$, which requires an uniform sample temperature during measurement. Measuring the sample after cooling to $77^{\circ} \mathrm{K}$ introduces a drift due to changing temperature of the specimen. To overcome this problem, we designed a special all-glass Dewar flask (double-walled, un-silvered) that is placed into the SI2B coil. This arrangement allows measuring the magnetic susceptibility of a sample while it is immersed in liquid nitrogen. The SI2B coil unit is placed vertically, so the glass dewar rests inside the coil. A thin jacket of styrofoam is placed around the Dewar to protect the coil from excessive cooling.

[13] The AMS tensor is determined from a six-orientation scheme, measuring every position twice $(\mathrm{M}=6, \mathrm{~N}=2)$. This scheme takes the same time as $\mathrm{M}=12$ and $\mathrm{N}=1$, but gives more precise principal susceptibilities [Stupavsky, 1984]. One of the advantages of the SI2B meter is that it determines magnetic susceptibility by measuring the coil without the sample and then again the induction with the sample. Thus, any diamagnetic contribution of the glass flask and liquid nitrogen is fully compensated.

[14] The high frequency of the SI2B coil responds to water because of its high dielectric constant (around 80). Liquid nitrogen has a dielectric constant of 1.4 and thus the capacitance of the Dewar containing liquid nitrogen does not cause spurious change in coil frequency.

[15] We test the suitability of the LT-AMS method using samples of three different rock types (siltstones, slates and 

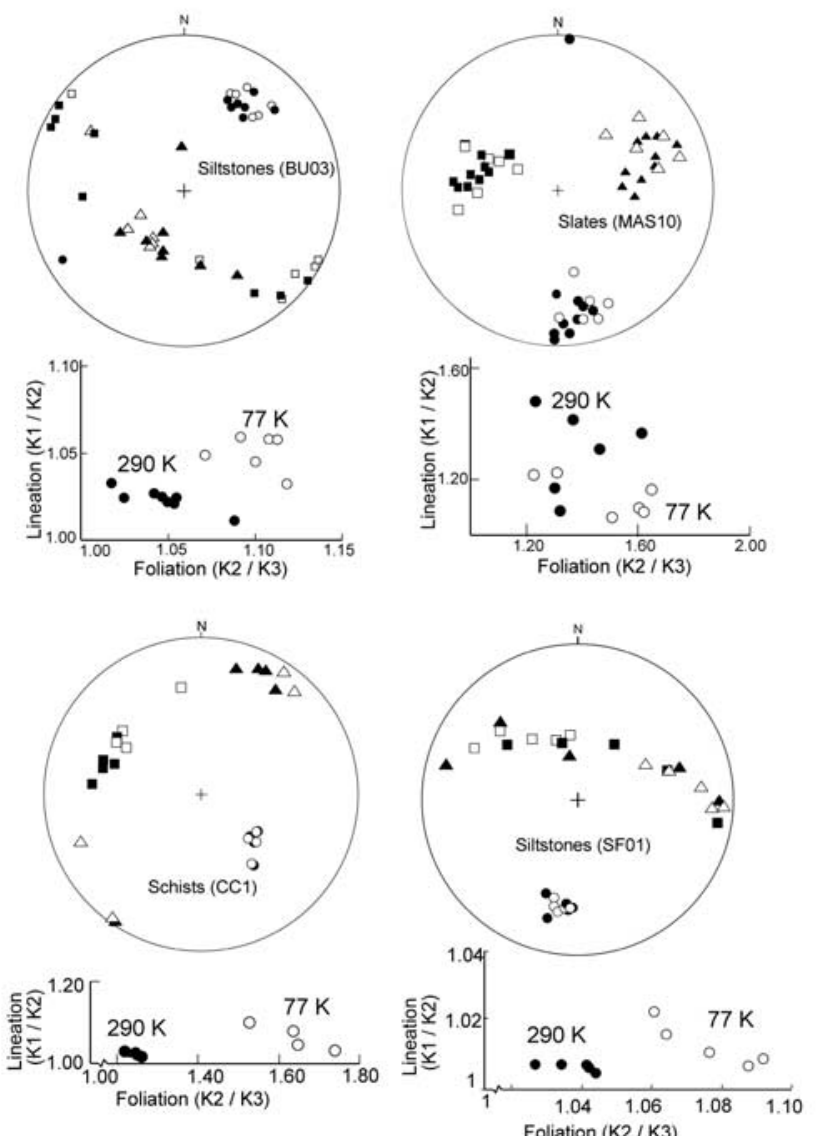

Figure 2. Principal magnetic susceptibility axes measured at room and liquid nitrogen temperature. See text for details of the procedure. Squares, triangles and dots represent maximum, intermediate and minimum principal axes of susceptibility respectively. Solid (open) symbols represent measurements at room (liquid nitrogen) temperature.

schists), which have distinct degrees of anisotropy. For each rock type, the AMS was measured both at room and liquid nitrogen temperature (Figure 3). That is, the orientation of the magnetic ellipsoid remains constant, suggesting that the magnetic anisotropy is mostly due to paramagnetic minerals. If a small component of ferromagnetic grains contributed to the AMS, they have a similar magnetic ellipsoid orientation as the paramagnetic ellipsoid.

[16] Whereas the axes orientations do not significantly change at $77^{\circ} \mathrm{K}$, the magnetic ellipsoid magnitude changes dramatically (Figure 2). The enhancement of the paramagnetic susceptibility at low temperature is reflected by an increase of the eigenvalues of the susceptibility tensor. Changes in eigenvalues are unequal and hence modify the ellipsoid eccentricity as shown in a Flinn-type diagram (Figure 2). In almost all cases, the magnetic ellipsoid becomes more oblate at $77^{\circ} \mathrm{K}$, with only a slight, if any, increase in magnetic lineation; that is, the thermal enhancement of magnetic susceptibility is anisotropic as $\mathrm{K}_{\max }$ increases by a factor larger than $\mathrm{K}_{\text {min }}$. The fact that susceptibility of iron bearing sheet silicates is more anisotropic at low temperatures has been previously noted for individual grains [e.g. Ballet and Coey, 1982]. For example, biotite has a marked dual magnetic structure, showing ferromagnetic along its basal plane, but the interactions across it are antiferromagnetic. The paramagnetic Curie temperature $\theta$ as measured along and perpendicular to the basal plane in biotite have markedly different values $\left[44^{\circ} \mathrm{K}\right.$ and $\left.-4^{\circ} \mathrm{K}\right]$ along and across the c-axis, respectively; [Beausoleil et al., 1983]. Similar observations have been made in iron-bearing phyllosilicates [Ballet and Coey, 1982; Ballet et al., 1985]. The underlying reason is that at low temperatures, when the susceptibility becomes sufficiently large, spontaneous ferromagnetic order appears within the plane of the sheets and not across them. We propose that these intrinsic changes in the anisotropy of susceptibility at low temperature can be used for clay and fabric characterization as different phyllosilicates have distinct Curie paramagnetic temperature $\theta$. The magnetic anisotropy for chlorite, muscovite and biotite specimens were measured at room and $77^{\circ} \mathrm{K}$ temperature (Figure 3). These three phyllosilicates have similar anisotropy degree $\left(\mathrm{K}_{\max } / \mathrm{K}_{\min }\right)$ at room temperature (small dots), but the magnetic anisotropy degree at $77^{\circ} \mathrm{K}$ is increased due to the disproportionate growth of $\mathrm{K}_{\max }$ and $\mathrm{K}_{\text {min }}$ (large dots). The ratio $\mathrm{K}_{\max } / \mathrm{K}_{\min }$ barely changes for chlorite, but increases from 1.1 to 1.8 for biotite.

[17] The results from siltstones, shales and schists samples (Figure 3a) show what kind of phyllosilicate dominates the susceptibility tensor, as the maximum $\mathrm{P}$ value attainable at $77^{\circ} \mathrm{K}$ for a given rock sample cannot exceed the $\mathrm{P}$ value of the phyllosilicate that carries the magnetic susceptibility. Hence, the value of magnetic anisotropy degree $\mathrm{P}$ at low temperature indicates the dominant phyllosilicate phase in the rock specimen. Given that the magnetic anisotropy increases at $77^{\circ} \mathrm{K}$, LT-AMS provides a high definition of relative grain preferred orientation in a rock. Grain re-orientation and its effect on the magnetic anisotropy is simulated by the anisotropic properties of a single crystalline grain, derived from a summation of the orientations of individual grains [see Owens, 1974, Tarling and Hrouda, 1993 and Hrouda and Schulmann, 1990 for details]. Therefore the degree of magnetic anisotropy of a rock whose anisotropy is dominated by a single mineral, provides a quantifiable estimate of the degree of mineral alignment [Hrouda and Schulmann, 1990; Richter, 1992; Tarling and Hrouda, 1993].

\section{Conclusions}

[18] The magnetic susceptibility of phyllosilicate-bearing rocks at liquid nitrogen temperature, due to paramagnetic behavior, is 3 to 5 times higher than at room temperature. In these cases, the intrinsic susceptibility becomes more anisotropic at low temperature due to ferromagnetic coupling within sheets. LT AMS allows a better definition of the degree of mineral alignment due to the enhancement of the magnetic anisotropy below room temperature (Figure $3 b$ ). In the studied samples, including siltstones, shales and schists, the LT-AMS ellipsoid has the same orientation as that at room temperature, confirming that the anisotropy tensor reflects the paramagnetic contribution. LT-AMS enhances fabric characterization in rocks where both ferromagnetic and paramagnetic grains compete to determine the AMS ellipsoid or when the paramagnetic signal is weak and poorly defined. In addition to providing a method for enhancing the paramagnetic contribution, the LT AMS methods offer a powerful, 
(a)

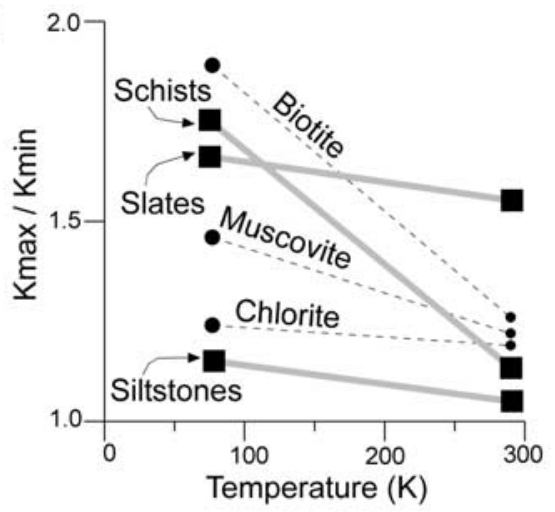

(b)

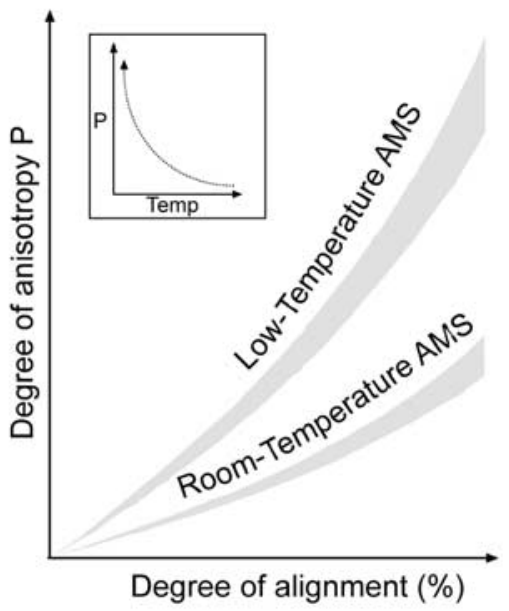

Figure 3. (a) Magnetic susceptibility changes of magnetic anisotropy degree $\mathrm{P}\left(\mathrm{K}_{\max } / \mathrm{K}_{\min }\right)$ as a function of temperature. Samples of biotite, muscovite and chlorite are shown as dots. Squares show samples of siltstones, slates and schists. Increase in anisotropy degree of the selected rocks reflects the dominant phyllosilicate phase. (b) Conceptual single-mineral model of rock magnetic anisotropy versus degree of mineral alignment. The degree of crystalline alignment can be directly determined from the degree of magnetic anisotropy $\mathrm{P}$ as indicated by the paths shown on the plot.

non-destructive approach to characterizing phyllosilicate fabrics in common terrigenous rocks.

[19] Acknowledgments. This work was supported by NSF Grant EAR9814343. The authors would like to thank L. Holladay for patiently carrying out some of the laboratory measurements. Reviews by John Geissman and an anonymous reviewer improved the manuscript.

\section{References}

Bergmuller, F., et al., A torque magnetometer for measurement of the highfield anisotropy of rocks and crystals, Meas. Sci. Technol., 5, 14661470, 1994.

Borradaile, G., and F. Lagroix, Thermal enhancement of magnetic fanrics in high grade gneisses, Geophys. Res. Lett., 27, 2413-2416, 2000.

Borradaile, G., and T. Werner, Magnetic anisotropy of some phyllosilicates, Tectonophysics, 235, 223-248, 1994

Borradaile, G. J., and B. Henry, Tectonic applications of magnetic susceptibility and its anisotropy, Earth Sci. Rev., 42, 49-93, 1997.

Ballet, O., and J. M. D. Coey, Magnetic properties of sheet silicates; 2:1 Layer minerals, Phys. Chem. Minerals, 8, 218-229, 1982.

Ballet, O., J. M. D. Coey, and K. J. Burke, Magnetic properties of sheet silicates; 2:1:1 Layer minerals, Phys. Chem. Minerals, 12, 370-378, 1985.
Beausoleil, N., P. Lavallee, A. Yelon, O. Ballet, and J. M. D. Coey, Magnetic properties of micas, J. Appl. Phys., 54, 906-915, 1983.

Dunlop, D., and Ozdemir, Rock Magnetism: Fundamentals and frontiers, Cambridge University Press, 1997.

Ferré, E. C., et al., Ductile flow in migmatites deduced from combined lowand high-field AMS measurements, Eos Transactions, AGU, 81(48), F367, 2000.

Housen, B. A., and B. A. van der Pluijm, Slaty cleavage development and magnetic anisotropy fabrics (AMS and ARMA), Jour. Geophys. Res., 96, 9937-9946, 1991 .

Hrouda, F., Magnetic anisotropy of rocks and its application in geology and geophysics, Geophysical Surveys, 5, 37-82, 1982.

Hrouda, F., and V. Jelinek, Resolution of ferrimagnetic and paramagnetic anisotropies in rocks, using combined low field and high field measurements, Geophys. J. Int., 103, 75-84, 1990.

Hrouda, F., and Schulmann, Conversion of the magnetic susceptibility tensor into the orientation tensor in some rocks, Phys. Earth Planet. Int., 63 , $71-77,1990$.

Ihmlé, P. F., et al., Inverse fabric in deformed limestones of the Morcles Nappe, Switzerland, Geophys. Res. Lett., 16, 1383-1386, 1989.

Jover, O., et al., Magnetic mineralogy of some granites from the French Massif Central: origin of their low-field susceptibility, Phys. Earth Planet. Int. 55, 79-92, 1989.

Kent, D. V., and W. Lowrie, On the magnetic susceptibility anisotropy of deep-sea sediment, Earth Planet. Sci. Lett., 28, 1-12, 1975.

Lüneburg, C. M., S. A. Lampert, H. D. Lebit, A. M. Hirt, M. Casey, and W. Lowrie, Magnetic anisotropy, rock fabrics and finite strain in deformed sediments of SW Sardinia (Italy), Tectonophysics, 307, 51-74, 1999.

Martín-Hernández, F., and A. M. Hirt, Separation of ferrimagnetic and paramagnetic anisotropies using a high-field torsion magnetometer, Tectonophysics, 337, 209-221, 2001.

Morrish, A. H., The physical principles of magnetism, Wiley, New York, 680 pp., 1965

Moskowitz, B. M., et al., Low-temperature magnetic behavior of titanomagnetites, Earth Planet. Sci. Lett., 157, 141-149, 1998.

Nye, J. F., Physical Properties of Crystals, Oxford Univ. Press, London, 322 pp., 1957.

Owens, W. H., Mathematical model studies on factors affecting the magnetic anisotropy of deformed rocks, Tectonophysics, v. 24, 115-131, 1974.

Parés, J. M., B. van der Pluijm, and J. Dinares-Turell, Evolution of magnetic fabrics during incipient deformation of mudrocks (Pyrenees, northern Spain), Tectonophysics, 307, 1-14, 1999.

Parés, J. M., et al., Low-Temperature Anisotropy of Magnetic Susceptibility (LT-AMS), Eos Transactions, $A G U, 81(48), \mathrm{F} 367,2000$.

Parma, J., An automated torque meter for rapid measurement of high-field magnetic anisotropy of rocks, Phys. Earth Planet. Int., 51, 387-389, 1988

Perarnau, A., and D. H. Tarling, Thermal enhancement of magnetic fabric in Cretaceous sandstone, J. Geol. Soc. Lond., 142, 1029-1034, 1985.

Richter, C., Particle motion and the modelling of strain response in magnetic fabrics, Geophys. J. Int., 110, 451-464, 1992.

Richter, C., and B. A. van der Pluijm, Separation of paramagnetic and ferrimagnetic susceptibilities using low temperature magnetic susceptibilities and comparison with high field methods, Phys. Earth Planet. Inter. 82, 113-123, 1994

Rochette, P., and G. Fillion, Identification of multicomponent anisotropies in rocks using various field and temperature values in a cryogenic magnetometer, Phys. Earth Planet. Int., 51, 379-386, 1988.

Schultz-Krutisch, T., and F. F. Heller, Measurement of magnetic susceptibility anisotropy in Buntsandstein deposits from southern Gremany, Journal of Geophysics, 56, 51-58, 1985.

Stupavsky, M., Operating manual for the SI-2 Magnetic susceptibility Instrument. Sapphire Instruments, PO Box 385, Ruthven, Ont., NOP 2GO, Canada, 1984.

Tarling, D. H. and F. Hrouda, The magnetic anisotropy of rocks, Chapman and Hall, London, 217 pp., 1993.

Thill, J. W., et al., Separation of AMS into ferromagnetic and paramagnetic components in migmatites: a possible shear-sense indicator?, Eos Transactions, $A G U, 81(48), \mathrm{F} 367,2000$.

Urrutia-Fucugauchi, J., and D. H. Tarling, Paleomagnetic properties of Eocambrian sediments in northwestern Scotland: implications for world-wide glaciation in the Late Precambrian, Paleogeog. Paleoclim. Paleoecol., 1, 325-344, 1983.

$\mathrm{Xu}, \mathrm{T}$. C., S. J. Ye, and F. Yang, A preliminary study of thermally enhanced magnetic fabric in the Tertiary sediments from the Qidam Basin, NW China, Stud. Geoph. Geol., 35, 295-301, 1991.

J. M. Pares and B. A. van der Pluijm, Dept. of Geological Sciences, University of Michigan, 2534 C.C. Little Building, Ann Arbor, MI 48109, USA. (jmpares@umich.edu) 